\title{
Computer Assisted Instruction in Teaching of Mathematics
}

\author{
Ms. Pramila Ramani ${ }^{1}$, Prof. Dr. Harsha Patadia ${ }^{2}$ \\ ${ }^{1}$ (Science and Education, Navrachana University, India) \\ ${ }^{2}$ (Education, MS University of Baroda (CASE), India)
}

\begin{abstract}
Mathematics is an abstract subject and symbol occupies an important position. Students find it difficult to comprehend this subject. There are many failures in mathematics than any other subject. Considering student teacher ratio and heterogeneous group of students in a normal classroom it is very difficult for a teacher to reach to all the students. Hence there is a need of supplement along with classroom teaching. Computer assisted instruction plays very important role in this situation. This paper explains the nature and importance of mathematics and Computer assisted instruction and its importance.
\end{abstract}

Keywords - Abstract, Computer Assisted Instruction, Heterogeneous Groups, Individualized Instruction,

Special Language

\section{INTRODUCTION}

"Education has always been important but perhaps, never more so, in man's history than today. In a science based world, education and research are crucial to the development process of a country, its welfare, progress and security" [1]

This statement is more relevant even after forty-six years in today's world of knowledge explosion but rather increased. The $21^{\text {st }}$ century world can be called a scientific world, advancing rapidly in information technology, medicine, engineering, space communication, astronomy, astrophysics, artificial intelligence, robotics and many other disciplines. Our country requires technically skilled manpower. For all disciplines mathematics is the base. The syllabus of mathematics at secondary level that is currently in use has been prepared by NCERT and is known as new pattern syllabus.

Mathematics is an abstract subject. The reasoning in mathematics possesses a number of characteristics, namely, characteristics of accuracy, verification of results, certainty of results, similarity to reasoning in life, originality. All these characteristics automatically become a part and parcel of a child when he learns mathematics. Mathematics has a special language in which symbol occupies an important position. Students find it difficult to understand mathematics because of symbols and abstractness. [2] Patel in her study specifies that one of the reasons for the selection of commerce stream was that students felt science stream to be difficult, as it requires a lot of hard work to be put in.

The Education Commission (1964-66) recommended mathematics as a compulsory subject for all school students. Thus, mathematics enjoys a unique status in a school curriculum. [3] The National policy on Education NPE- 1986 also emphasizes that mathematics should be visualized as the vehicle to train a child to think, reason, analyze and articulate logically, apart from being a specific subject it should be treated as concomitant to any subject involving analysis and reasoning. Yet many school students find difficulty with learning of mathematics and fail in mathematics. A major reason for the failure is that the teachers quite often pay no attention to the basic concepts and generally adopt methods of solving questions with crammed up formulae. [4] "Ours and previous few generations have failed to produce good mathematics teachers at school level in adequately large numbers." The corpus of this enormous knowledge that man built over the last few centuries will be too burdensome to carry into future on the shoulders of ill-equipped school Mathematics teachers. This is so since teaching mathematics to impressionable young minds is a specialized task that many mathematicians may not measure. Mathematics is a hard task master that demands implicit and whole attention from the disciple.

In order to overcome the difficulties faced by the students, teacher should adopt different methodology in teaching of mathematics like drill method, using different audio visual aids, computer aided instruction, mathematical club etc. One of the methods is auto-instructional method. It is a method of individualized instruction. One of its forms is CAI (Computer Assisted/Aided Instruction) auto instructional teaching. This aspect is elaborated in topics that follow.

1.1 Importance of Mathematics

Epistemologically mathematics means mathema- explaining and understanding, tics- techniques such as counting, ordering, sorting, and measuring. Right from pre historic period there have been problems to solve. Problems may be over basic requirements like food, water, shelter or accomplishment like constructing multi- 
storied building. In this modern era we cannot think of a field, where calculation or computation is not used. Knowingly or unknowing we use mathematics in our day-to-day life. It ranges from household to industries, business, education, science and technology, art and craft and even in music, dance etc.,

\subsection{Nature of Mathematics}

Mathematics relies on both logic and creativity, and it is pursued both for a variety of practical purposes and for its intrinsic interest. Language of mathematics is symbolic and less verbose. Mathematics is the science of patterns and relationships.

[5] "Mathematics is the science of number and space. Mathematics is the science of measurement, quantity and magnitude. Mathematics is also called science of logical reasoning. Mathematics may also be defined as the science of abstract and imaginative form."

Education Commission (1964-65) "One of the outstanding characteristics of science culture is qualification of Mathematics".

- Mathematics is hierarchical in nature.

- Mathematics is science of logical reasoning.

- Mathematics is more than computation

- Mathematics has peculiar language and symbolism. It has a different language and syntax and uses many words from day today life but in different sense.

- Mathematics is abstract in nature.

According to [6] characteristics of Mathematics is listed as follows:

- It is the science of number and space.

- It is the science of calculation.

- It is the science of measurement, quantity and magnitude.

- It is systemized, organized and exact branch of science.

- It deals with quantitative facts and relationship.

- It is the abstract form of science.

- It is science of logical reasoning.

- It settles in the mind the habit of reasoning

\section{CAI IN LEARNING MATHEMATICS}

ICT (Information and Communication Technology) has great potential for teaching and learning process at all levels. The use of ICT has enriched the teaching learning process with the help of computer. It has brought a great change, innovativeness, and creativity in teachers in teaching learning process. Mathematics and computer are both important in today's life as they open the gate of ample opportunities in this modern world. Mathematics is widely used in computers both in hardware and software. Computer helps in improving the knowledge of mathematics. Computer helps in making classroom teaching lively.

Computer can play vital role in learning process as it can work with the imagination of students. Any concept in mathematics can be explained with the help of pictures and this visual image can help in understanding the concept at ease. In paper pencil method student can get bored easily and can find it difficult to practice the sum again and again. CAI works as a change and increases the curiosity of students and they can learn interestingly without any difficulty. Also whatever is learnt through computer aided instructions, the contents can be retained for longer time as they use more senses of the students. Certain chapters like Profit and loss, Simple and compound interest can be explained very easily using CAI. Varity of exercises can be provided and this ensures active involvement of the students. The material can be provided according to the needs of the students.

\subsection{Advantages of CAI in learning Mathematics}

In this age of technology the teacher should be aware of student's need. CAI can help to satisfy the needs of the students. CAI lessens the workload of the teachers, besides it has many other advantages as given below

- Provides Wide Range Of Experiences

CAI helps the teacher to provide a wide range of experience s/he can give many examples and illustrations and can make the concept clear.

- Provides Motivation

It can sustain the motivation of the students as the topic can be presented in an enjoyable manner as concepts can be presented systematically, interestingly and immediate feedback can be given which sustains the motivation of the students. Graphics and pictures can be presented which can attract and retain student attention. Children get reinforcement when they answer the question correctly and the topic is presented in a systematic manner in an increasing order of difficulty. 
- Individualized Instruction

CAI is an individualized instruction as it caters to the individual difference. Some students are slow learners and some are fast learners. The Indian classroom is a heterogeneous group. Some students need more time to learn while others need less time, so learning speed differs from learner to learner. CAI also provides different learning experience according to the understanding level of the students. It also provides facilities like selecting the topics of their own interest. It provides individual attention to each and every student and thus enhances the quality of teaching learning process and thus we can overcome the problems faced in a overcrowded classroom.

- Interactive Learning

CAI provides immediate feedback to the students and thus constantly interacts with them. In CAI students actively take part in the learning process. As it contains many examples and diagrams it makes the learning process interesting.

1.2 Principles of CAI

Principle of CAI is same as PLM

- Principle of small steps

- Principle of active responding

- Principle of reinforcement

- Principle of self-pacing

- Principle of student -evaluation or student testing

\subsection{Characteristics of CAI}

CAI refers to any use of computers that interacts with students in any way in the educational process.

- Practice: CAI enables the students to practice as many times as they like so this will enable them to achieve the required competencies. Students come from different background it is a heterogeneous group so their understanding level differs from student to student so a single teacher cannot cater such heterogeneous group so there is a need of right learning tool and a supporting environment. Practice makes a man perfect. Many psychologists like Thorndike support the usefulness of practice in learning.

- Immediate feedback: CAI enables the students to see the correct answer immediately as soon as they answer a particular question so that they can correct themselves. If the answer is correct then they will get immense happiness and added confidence. If the answer is wrong they can correct themselves immediately. In traditional classroom teaching, teacher gives students homework for practice. The child comes to know of any mistake when the teacher checks the homework and corrects the mistake. Normally teachers do not provide the correct answer during checking, so child knows that his answer is wrong but does not know the correct answer. If the teacher does sometimes provide the correct answer, the child may not pay due attention to the corrected answer and may consider it as a part of his work is to complete the homework and would proceed with the next homework.

- Self-Evaluation: CAI enables the students to find their strengths and weakness and student can overcome his weakness before proceeding further.

- Reinforcement: CAI reward students immediately whenever they answer the question correctly immediate reinforcement gives immense pleasure as indicated by many psychologists.

- Immediate Evaluation: As soon as each concept is completed students should answer questions related to that particular concept this enables immediate evaluation.

\section{Limitations Of Preparing CAI}

- Constructing a program needs a highly specialized skill.

- The author should have thorough knowledge of the subject matter and of programming techniques.

- The steps should proceed in a more sequential and illustrative examples should be selected carefully so that error-rate of a learner has to be minimized. If the learner commits more error then he will be de motivated.

- It consumes more time.

- To achieve mastery learning

\section{NECESSITY OF CAI}

Mastery is a recent innovation introduced in the sphere of education. Mastery learning implies a systematic approach to the process of teaching or instruction.

- Individual differences 
Each and every child is different. In a classroom teacher teaches to a group of students. Some students are slow learners, some are medium, and some are gifted. Classroom group is a heterogeneous group they come from different family background and with different interest. Some are extrovert and some are introvert.

- To make learning continuous.

When the child is absent on the previous day he cannot understand the lesson taught on that day because of lack of continuity and whatever is taught on that day he finds difficult to comprehend. CAI helps him to understand the concept without any difficulty whenever he misses a class.

- Span of attention

The time span the student remains attentive in a class. This is different for different students.

Definition of attention

[7] "Attention may be described as the selective activity of the human organism whereby one's consciousness is focused upon a specific, narrow field to the exclusion of everything else in the environment."

\section{CONCLUSION}

In a typical mathematics classroom we can observe that teacher spends approximately 5 minutes for introduction, 15 to 20 minutes for content explanation, 10 to 15 minutes for question and answers to heterogeneous group's verification and confirmation either at the end or continuously along with teaching. Thereafter, the teacher recapitulates all the subtopics. It is not possible to interact with all students, so teacher interacts with about 4 to 5 students. Having confirmed that these 4 to 5 students have understood the content explanations, the teacher assumes that the entire class has followed the content. Some students are mere spectators because they did not get a chance to answer. In this process teacher cannot cater to slow learners, obviously the teacher does not have time. This is not the teacher's fault but the system's fault. Certain students cannot grasp the contents fully. So it is not possible for the teacher to cater to all the students. Certain methodology and supporting methods are needed hence there is a need for development of CAI in mathematics. Certainly students cannot learn the entire topics by themselves using CAI, teachers roll is very important therefore CAI can be used as a supplement along with the teachers teaching.

\section{REFERENCES}

[1] NCERT (1971) Education and National Development. Report of the Education Commision1964-66. New Delhi: NCERT

[2] M.E .Patel, Factors Influencing Higher Secondary Students for Selection of Commerce and Rejection of Science Stream in Vadodara City: An Unpublished M.Ed Dissertation, Vadodara: CASE, The Maharaja Sayajirao University of Baroda.Vadodara, 2006.

[3] NPE (1986-1992). Reorienting the content and process of education. Part VIII in http://www.educationforallinindia.com/page57.html

[4] G. Ravindra, Researches on Curriculum and teaching:Mathematics: A Trend Report. in NCERT(Ed.) Sixth Survey of Educational Research 1993-2000, Vol.II, NCERT. New Delhi, 2006.

[5] K.L. Sidhu, The teaching of Mathematics. New Delhi: Sterling Publishers Private Limited, 1995.

[6] NCERT. Position Papers of National Focus Groups on Curricular Areas Volume I.NCERT New Delhi, 2005.

[7] S.Dandapani, A textbook of Advanced Educational Psychology. New Delhi Anmol Publication, 2001. 